\title{
Using Anti-Protons to Measure Nucleon Structure - Prospects at PANDA
}

\author{
B. Seitz on behalf of the PANDA collaboration \\ University of Glasgow - Department of Physic and Astronomy \\ Kelvin Building, Glasgow G12 8QQ - Scotland, U.K.
}

\begin{abstract}
Fundamental questions of hadron and nuclear physics can be addressed in interactions of anti-protons with nucleon and nuclei. Proton-antiproton annihilation is a unique tool to explore the structure of the nucleon in the time-like domain and to access quark distribution functions using Drell Yan processes or hard exclusive reactions. The planned Facility for Antiproton and Ion Research (FAIR) featuring a high energy storage ring for anti-protons will deliver a high quality beam ideally suited to access this physics with unprecedented accuracy. A universal detector system called PANDA is proposed to surround an internal target covering almost the complete solid angle.
\end{abstract}

\section{Introduction}

FAIR is a major upgrade planned at the existing GSI facility at Darmstadt. It covers a very broad range of modern hadronic physics. The physics program with anti-proton beams covers the investigation of hadrons and the properties of hadronic matter in the energy range up to $\sqrt{s} \approx 5.5 \mathrm{GeV}$ and at luminosities in excess of $\mathcal{L}=10^{31} \mathrm{~cm}^{-2} \mathrm{~s}^{-1}$. The main topics to be addressed are the search for charmed hybrids and glueballs, spectroscopy of charmonium, the study of hypernuclei as well as a measurement of the properties of the proton in reaction with electromagnetic final states.

The annihilation of anti-protons with protons can be used to probe the structure of the nucleon itself by looking at electromagnetic final states, i.e. reactions with real or virtual photons as reaction products. The most prominent reactions discussed in the following are the study of parton distribution functions in the Drell Yan process and the study of the time-like behaviour of so called Generalized Distribution Amplitudes in exclusive processes and the time-like behaviour of the protons electromagnetic form factors.

The broad physics program to be addressed by this new facility poses significant challenges for the design and construction of the detector. In particular, the detector should provide:

- full angular coverage and good angular resolution for charged and neutral particles

- particle identification in a large range of particles and energies

- high resolution over the required wide range of energies

- high rate capability especially for the close-to-target and forward detectors.

The PANDA experiment thus is a multi-purpose detector system surrounding an internal target. In the following several highlights focussing on the measurement of electromagnetic final states will be briefly described, more details can be found in Ref. [2].

\section{The PANDA experiment}

The detector design is shown in Fig. 1. Its concept is based on a combination of a central detector (target spectrometer (TS)) within a $2 \mathrm{~T}$ solinoidal magnetic field covering labora- 
tory angles from $5^{\circ}$ on upwards complemented by a forward spectrometer (FS) equipped by a dipole magnet to measure particles at the most forward angles. Combining these two spectrometers allows full angular coverage and the wide range of energies to be taken into account. Both, TS and FS, will allow the detection, identification and energy/momentum reconstruction for charged and neutral particles. Particles emitted with laboratory angles

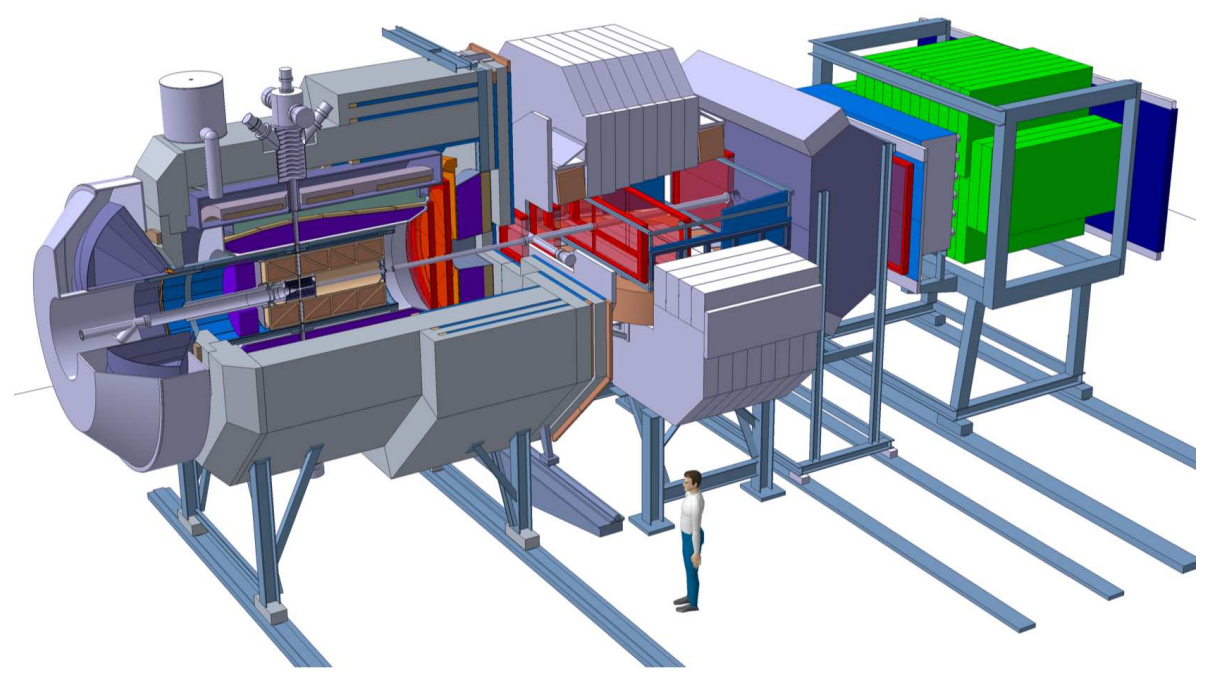

Figure 1: Sketch of the planned PANDA detector systems. The antiproton beam is entering the target spectrometer from the left. The forward spectrometer is depicted on the right.

larger than $10^{\circ}$ are solely measured in the TS consisting of azimuthally symmetric detectors in the coils of a super-conducting magnet. Surrounding the interaction volume will be a silicon micro-vertex detector. This detector is followed by the central tracking detector, e.g a TPC. Particle identification will be realised by a variety of different techniques. The main work horse in the TS will be a DIRC-type Cherenkov counter complemented by Time-of-Flight systems and measurements of the energy deposited in the TPC and the electromagnetic calorimeter. The forward region will be covered by two sets of mini drift chambers and a flat disc DIRC. Muon-detectors will be mounted outside of the return yoke. Particles emitted with polar angles below $10^{\circ}$ horizontally and $5^{\circ}$ vertically are detected with the FS. It is comprised of a $1 \mathrm{~m}$ gap dipole and tracking detectors for momentum analysis of charged particles. Photons will be detected by a shashlyk-type calorimeter consisting of lead-scintillator sandwiches. Other neutrals and charged particles with momenta close to the beam momentum will be detected in the hadron calorimeter and muon counters. Identification of charged particles will be provided by Cherenkov and ToF counters.

\section{Electromagnetic final states at PANDA}

Anti-proton - proton annihilation reactions yielding electromagnetic final states probe the nucleon structure in the time-like region. They are complementary deep inelastic scattering 
in probing the underlying parton distributions. Choosing a $\bar{p}$-beam allows in particular to study annihilation in the valence quark region. PANDAs excellent detector capabilities allow to separate and identify rare events with only two real photons, a real and a virtual photon (or meson) or a lepton pair in the final state offering a unique possibilities.

\section{Drell Yan processes}

The Drell Yan processes has evolved to be a standard tool for probing the partonic structure of hadrons. PANDA offers the unique opportunity to measure these processes in $\bar{p} p$ annihilation probing not only the sea-quarks, but more importantly the valence quark region. The angular dependence of the Drell Yan cross section is given by:

$$
\frac{d \sigma}{d \Omega} \propto 1+\lambda \cos ^{2} \theta+\mu \sin 2 \theta \cos \phi+\frac{\nu}{2} \sin ^{2} \theta \cos 2 \phi .
$$

At NLO, the parameters $\lambda$ and $\nu$ are related by the Lam-Tung relation $1-\lambda=2 \nu$ [3], which was shown to be violated in pion-induced Drell Yan processes at high $p_{T}$. This violation has been explained e.g. by the existence of a chiral-odd, T-odd distribution function with an intrinsics $k_{T}$ dependence [4]. This hitherto unmeasured Boer-Mulders function represents the correlation of the parton's transverse spin and $k_{T}$ in an unpolarised nucleon. The Boer-Mulders function belongs to a class of functions which recently generated considerable interest, with e.g. the Collins fragmentation function, the transversity distribution and the Sivers function belonging to the same family. The latter two functions will be uniquely accessible in doubly polarised or single polarised Drell Yan processes [5]. The Sivers function is expected to change sign between measuring it in semi-inclusive DIS and in Drell Yan reactions. The observation of this sign change would be a fundamental test of QCD.

\section{Time-like form factors}

The electric and magnetic form factors $G_{E}$ and $G_{M}$ describing the response of the proton to time-like photons can be extracted from the cross section of the process $\bar{p} p \rightarrow e^{+} e^{-}$. Both the electric and magnetic form factors depend on the virtuality of the intermediate photon $Q^{2}$. A measurement over a large range of $Q^{2}$ is thus crucial to test the $Q^{2}$-behaviour as well as the space-like time-like equality for each value of $Q^{2}$ measured. The proton time-like form factors have been measured by various experiments near threshold and in the region of low values of $Q^{2}$. The current upper range was pioneered by E760 and E835 at Fermilab reaching up to $Q^{2} \approx 15 \mathrm{GeV}^{2}$, while the intermediate range was recently covered by BES and BaBar [6, 7]. These experiments did not achieve the statistics required to separate $\left|G_{E}\right|$ and $\left|G_{M}\right|$ but had to work under the assumption of both being equal. A rich structure has been predicted using the scarce data in the time-like region and analytic continuation from the space-like region [8]. With PANDA running at design luminosity it will be possible to determine both form factors separately and over a large range in $Q^{2}$ reaching from threshold up to $Q^{2} \approx 20 \mathrm{GeV}^{2}$ or higher within a single experiment. The expected improvements in angular coverage and statistics compared to e.g. the previous experiments at Fermilab will significantly improve the existing results and allow a separation of $\left|G_{E}\right|$ and $\left|G_{M}\right|$. 
Hard exclusive reactions

The recently established framework of Generalised Distribution Amplitudes (GDA) (or Transition Distribution Amplitudes (TDA)) offers a new tool in understanding the structure of the nucleon unifying diverse probes of its structure like form factors, forward parton distributions, hard exclusive production or wide angle Compton scattering. It was shown recently that the exclusive $\bar{p} p$ annihilation at large $s$ and $t$ can be described in terms of GDAs as well. At these kinematics, the reaction separates into a soft part parametrised by GDAs and a hard part describing the annihilation of a quasi-free $\bar{q} q$-pair into two photons.

Data from a wealth of different reactions are needed to map out this new class of functions promising a complete picture of the nucleons' structure. Most of the theoretical attention was give to the process $\bar{p} p \rightarrow \gamma \gamma$, which offers access to GDAs without additional complication like the wave-function of the mesons produces. Based on the calculation of Ref. [9] a rate of a coupled of thousand of these events will be produced per month with PANDA running at design luminosity. In addition to this, the most simple process $\bar{p} p \rightarrow \gamma \gamma$ more complicated exclusive annihilation processes with (pseudo)scalar or vector mesons as well as virtual photons in the final state will be considered as well. Interpreted in terms of TDAs, this will allow e.g. to measure the deep spatial structure of quarks inside the nucleon [10]. Taking data from hard exclusive processes in exclusive deep inelastic scattering and $p \bar{p}$ together promises for the fist time to measure the spatial location of quarks inside a nucleon.

\section{Conclusion and outlook}

The PANDA experiment at FAIR will be a future key experiment in understanding the nature of QCD and the structure of the nucleon. PANDA combines a unique $\bar{p}$-beam at high luminosity and precision with an innovative, complete detector set-up. This unique combination will allow precision measurement of $p \bar{p}$-annihilation into electromagnetic final states. Parton distribution functions of the nucleon like the Sivers-function and the BoerMulders-function are directly accessible in Drell Yan processes. The measurement of hard exclusive reactions allows access to GDAs and thus ultimately to a complete understanding of the nucleon. Last not least, measurements of time-like nucleon form factors offer exciting prospects. The PANDA experiment at FAIR will address all these issues, promising a rich harvest of physics results.

\section{References}

[1] Slides:

http: //indico. cern. ch/contributionDisplay py? contribId=259\&sessionId=10\&conf Id=9499

[2] PANDA Collaboration, Technical Progress Report, FAIR-ESAC/Pbar 2005.

[3] C. S. Lam, W. K. Tung, Phys. Rev. D 212712 (1980).

[4] D Boer, Phys. Rev. D 60014012 (1999).

[5] P. E. Reimer, J. Phys. G: Nucl. Part. Phys. 34 S107 (2007).

[6] M. Ablikim et al. [BES Collaboration], Phys. Lett. B 63014 (2005).

[7] B. Aubert et al.[BaBar Collaboration], Phys. Rev. D 73012005 (2006).

[8] R. Baldini et al., Eur. Phys. J. C 46421 (2006).

[9] A. Freund et al., Phys. Rev. Lett. 90092001 (2003).

[10] B. Pire, L. Szymanowski, Nucl. Phys. A 7829 (2007). 